Journal of

Accident and

Emergency

Medicine 1994

11, 201-203

\title{
Aluminium ring pulls: an invisible foreign body
}

\author{
G.D.STEWART, M.V.LAKSHMI \& A.JACKSON
}

Department of Radiology, Department of Medicine and Department of Neuroradiology, Manchester Royal Infirmary, Oxford Road, Manchester

\section{SUMMARY}

The aluminium ring pulls associated with the latest designs of drinks cans can be relatively easily detached from their mounting on the top of the can and subsequently aspirated. Their small size predisposes them to lodge as foreign bodies (FBs) in the throat. The similarity of atomic number between soft tissue (7.5) and aluminium (13) makes detection of these F.Bs difficult on soft tissue radiography. If aspiration is suspected direct visualization and removal may be indicated even if radiography is negative.

Key words: aluminium, ring pulls, STV neck

\section{INTRODUCTION}

The introduction of aluminium drinks cans as an aid to recycling poses a new medical problem. Aluminium ring pulls can become detached from their mountings, fall into the can and be subsequently swallowed or inhaled. The exclusion of cervical FB clinically can be difficult. An FB lacerating or puncturing the soft tissues will give the subjective impression that the FB is stuck, even if it has passed distally. Diagnosis, therefore, relies on a combination of soft tissue neck radiography and indirect laryngoscopy. Indirect laryngoscopy can be difficult in some patients who are unable to tolerate the required instrumentation. Visualization of $F B$ on soft tissue views (STV) of the neck has recently been called into question with some fish bones being shown to be undetectable on STV of the neck. ${ }^{1}$ Although it might be thought that aluminium FBs would be easy to detect, similar factors militate against their detection on standard soft tissue radiography. Three cases of swallowed aluminium ring pulls are reported. Only one previous case of an oesophageal aluminium FB has previously been described. ${ }^{2}$

\section{Case one}

A 43-year-old man attended the ear, nose and throat (ENT) department complaining of minor irritation $\frac{\bar{D}}{3}$ below the cricoid after swallowing a ring pull from a Coca-Cola can. He denied any further symp- $\vec{\omega}$ tomatology. Chest and STV neck films showed no 오 evidence of FB or other abnormality and indirect $ᄋ$ laryngoscopy also failed to demonstrate an abnor- $\vec{c}$ mality. The patient was reassured and discharged. $\mathbb{D}$ The patient reattended 4 days later with cough, $\mathbb{\mathbb { D }}$ retrosternal pain radiating to the left arm and hae- $\frac{3}{\Phi}$ moptysis. He was also apyrexial. Repeat posteroanterior and lateral chest radiographs, emergency barium swallow and ECG were normal. The patient was again discharged. One month later, the patient was referred with a history of recurrent chest infections, haemoptysis and right upper chest pain $\stackrel{0}{\mathbb{D}}$ despite several courses of appropriate antibiotics. He underwent fibreoptic bronchoscopy and a ring pull was removed from the right lower lobe bronchus The patient recovered completely.

\section{Case two}

A 34-year-old man presented to the accident and emergency (A\&E) department after swallowing 윽 a ring pull from a beer can. Beyond some initial $D$ discomfort, he experienced no difficulty in breathing or swallowing, and had no persistent choking. On o examination there was no focal tenderness or $\stackrel{N}{N}$ evidence of drooling. Departmental chest film and $\mathrm{\omega}$ STV of the neck (Fig. 1) were considered normal and the patient was discharged. At follow-up the $\frac{0}{\mathscr{C}}$ patient reported that he had passed the ring pull in a stool 3 days after the first attendance. Review of the initial STV neck showed a doubtful FB anterior $\frac{\mathbb{D}}{\mathbb{D}}$ to the C6 vertebra. Subsequent STV of the neck $\stackrel{\square}{\mathbb{Q}}$ showed resolution of this shadowing (Fig. 2). The patient was, by this time, asymptomatic. 


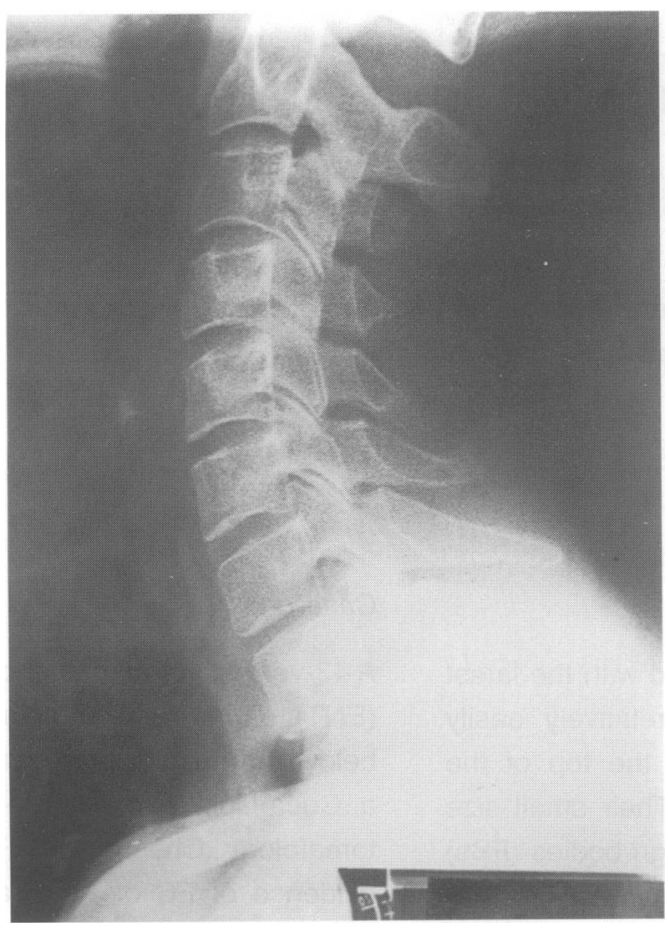

Fig. 1. STV of the neck in patient two at presentation showing a density anterior to the $\mathrm{C} 5 / 6$ disc space which was not appreciated at the time.

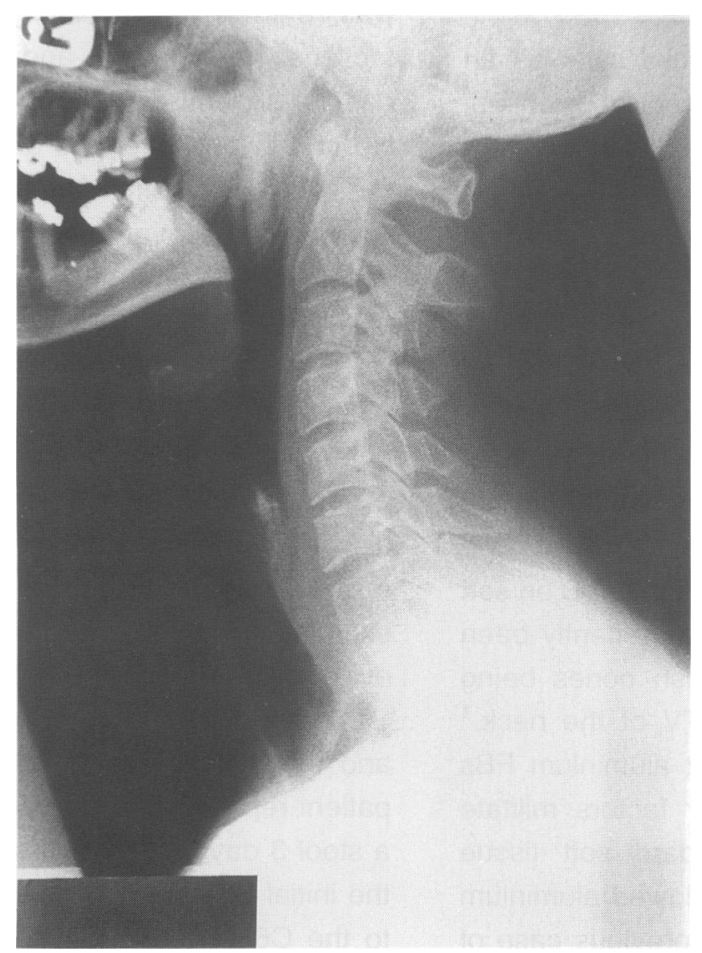

Fig. 2. Follow up film in the same patient showing no evidence of FB.

\section{Case three}

A 62-year-old man attended the A\&E department following ingestion of a ring pull from a Coca-Cola can. Films of chest, abdomen and STV of the neck showed no evidence of FB. Indirect laryngoscopy was normal and the patient developed no further symptoms. At follow-up he reported passage of the ring pull with no further symptoms.

\section{DISCUSSION}

The aim of modern radiographic imaging apparatus is to form an image of acceptable diagnostic quality while giving as low a dose as can be reasonably achieved (the ALARA principle). ${ }^{3}$ Radiographic contrast depends on the following three factors: (1) the character of the beam, (2) the density of the medium traversed and (3) the nature of the enregistration medium.

Although intuitively it would seem that a metal FB should be radio-opaque, all three of these factors militate against radiographic detection, partly because of attempts to reduce patient exposure and partly because of physical principles.

\section{Beam character}

The character of the beam is modified to reduce patient dose. Only $1 \%$ of the total energy applied to the X-ray tube is converted to X-ray photons, and the mean energy of these is only one-third to onehalf of the applied kilovoltage. Low energy photons are unable to penetrate the body and contribute to the image but if not removed would account for up to $80 \%$ of skin dose. The diagnostic quality of the beam is improved by aluminium filtration. Aluminium is utilized in beam filtration because of its $\mathrm{K}$-edge. The K-edge of a material depends on the binding energy of inner electrons which is proportional to the cube of the atomic number. Photon energy at and just above this ionization energy is statistically much more likely to cause this reaction, which selectively removes these photons from the beam. Recommended aluminium equivalent filtration for exposures of less than $70 \mathrm{KVP}$ is $1.5 \mathrm{~mm}$. The average exposure of an STV of the neck is approximately 50-60 KVP $10 \mathrm{mAs}$. Consequently, X-ray photons that would give maximum contrast between aluminium $Z=13$ and soft tissue $Z=7.5$ are selectively removed in formation of the beam. 


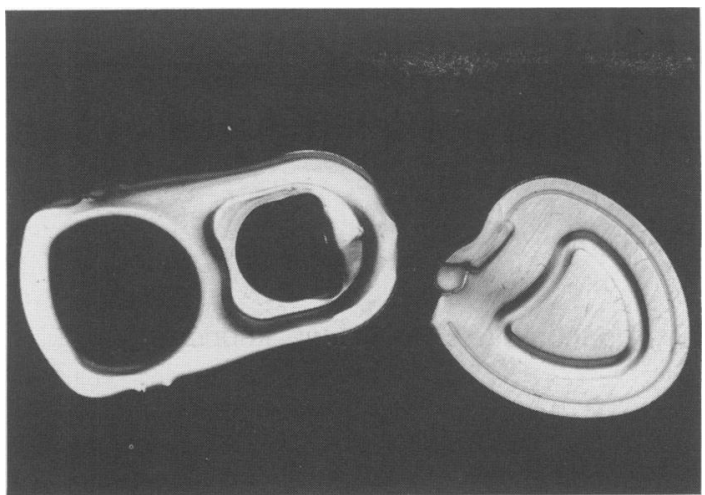

Fig. 3. View of aluminium ring pull showing detached distal fragment.

\section{Patient/beam interaction}

Within the body, contrast results from differential absorption of X-ray photons. In the diagnostic energy range, only photo-electric absorption - ionization with consequent loss of the $\mathrm{X}$-ray photon from the image - and Compton scatter related to the electron density of the material are of significance in forming the image. Photo-electric absorption (ionization) gives greater contrast as energy is completely absorbed within the tissues. Aluminium $(Z=13)$ is intermediate between bone $(Z=20)$ and soft tissue $(Z=7.5)$. The relatively small difference in atomic number and hence photo-electric absorption at soft tissue radiography voltages is compounded by removal of a disproportionate amount of that part of the beam close to the ionization energy.

\section{Film and observer limitations}

This effect is aggravated by the planar nature of the radiographic image. The dimensions of a ring pull are $20 \times 18 \times 0.5 \mathrm{~mm}$ (Fig. 3). En face the increase in total density across the width of the soft tissues of the neck where the pull is present, against where it is not, is trivial. If, however, the pull is oblique to the beam, any increased density on the image will be graduated, which the human eye finds difficult to distinguish because of retinal edge inhibition. It might appear that ring pulls orientated along the line of the beam would be most likely to be detected. Even here narrow angle Compton scatter, the other main mechanism of beam attenuation, will partially obscure the increased density.

Many of these radiographic problems are soluble at the expense of increased absorbed dose. Reduced filtration, lower KVP exposure and use of non-screen films would all help in visualization but would markedly increase dosage. Other radiological findings may be helpful on STV neck films where no FB is visible. There may be swelling of the prevertebral tissues with or without gas within the soft tissues. The prevertebral soft tissue anterior to $C 5 \frac{\mathrm{g}}{\mathrm{g}}$ should not be greater than half the vertebral body $\overrightarrow{0}$ width in an adult. However, swelling and soft tissue gas can be present after abrasion of the pharynx by FBs that have subsequently passed distally.

The problem may be avoided by modifying the manufacture of aluminium ring pulls. Previous dental $\dot{\omega}$ research ${ }^{4,5}$ has suggested the need for incorpor- $\stackrel{\text { N }}{\stackrel{0}{S}}$ ating radio-opaque material into dentures to facilitate $\frac{\rho}{9}$ recognition on radiographs. Similar modification of $\vec{D}$ ring pulls incorporating radio-opaque substances $\mathbb{D}$ would resolve this problem. Alternatively, adoption of the Japanese beer can where the ring can be pulled out rather than pushed in would help prevent ingestion.

In the meantime, plain film detection of aluminium ring pulls is unreliable and a high index of clinical suspicion supported by direct visualization at indirect laryngoscopy is suggested in those cases where either inhalation or swallowing is suspected.

\section{REFERENCES}

1. Ell S.R. \& Sprigg A. (1991) The radio-opacity of fishbones - species variation. Clinical Radiology 44, 104107.

2. Hewitt G. (1992) An aluminium foreign body in the oesophagus. Ulster Medical Journal 61 (1), 106-107.

3. ICRP (1977) Recommendations of the International Commission on Radiological Protection. Publication 26; Annals of the ICRP 1 (3). Pergamon Press, Oxford.

4. Payne S.D.W. \& Henry M. (1984) Radiolucent dentures impacted in the oesophagus. British Journal of Surgery 71 (4), 318.

5. Sansbury P. (1964) Radio-opaque denture resins. Dental Practitioner and Dental Records 14, 243-244. 